\title{
Evaluación de procesamiento auditivo y percepción sonora en sujetos con presbiacusia
}

\section{Ear predominance in presbycusis patients and auditory processing}

\author{
Mariela Torrente $\mathbf{A}^{1,2}$, Alexis Leiva $\mathbf{C}^{3}$.
}

\begin{abstract}
RESUMEN
Introducción: Pacientes portadores de hipoacusia simétrica en la audiometría pueden percibir su déficit como asimétrico. La explicación de este fenómeno podría tener su origen en variaciones en la conducción del estímulo hablado hacia la corteza auditiva, o procesamiento auditivo.

Objetivo: Evaluar el procesamiento auditivo en pacientes que refieren escuchar mejor por un oído en presencia de umbrales audiométricos simétricos.

Material y método: Pacientes mayores de 65 años. Criterios de exclusión: patología otológica, deterioro cognitivo (Mini Mental test <21 puntos), usuario de audífono, hipoacusia asimétrica. La batería de procesamiento auditivo incluyó evaluación de comprensión del habla en competencia (habla en ruido), pruebas dicóticas (dígitos dicóticos y la versión en español de Staggered spondaic words, SSW), y evaluación temporal (patrón de frecuencia y discriminación de silencio). Todas estas pruebas estaban previamente validadas en población chilena. Análisis estadístico mediante programa SPSS. Se utilizaron estadísticos descriptivos y pruebas no paramétricas (Kruskal Wallis y Mann Whitney). $p<.05$. Protocolo aprobado por comité de ética de Investigación, pacientes consienten. Trabajo financiado por Concurso de Proyectos de Investigación de la SOCHIORL y Proyecto Anillo ACT1403.
\end{abstract}

Resultados: 49 pacientes cumplen criterios de ingreso, 37 son de sexo femenino. Se identifican tres grupos: escuchan mejor por oído derecho (n: 19), por oído izquierdo (n: 14), o sin diferencia entre ambos oídos (n: 16). El grupo de pacientes con audición simétrica tenía PTP significativamente menor que los otros dos grupos. Al analizar los distintos tests de procesamiento auditivo no hay diferencia entre los grupos. Al evaluar habla dicótica se observó que el oído derecho tuvo un significativo mejor rendimiento que el oído izquierdo para dígitos dicóticos y SSW (p .000 y .007 respectivamente, test de Mann Whitney) en todo el grupo de pacientes evaluados, sin diferencia al agrupar por percepción auditiva (p .835, test de Kruskal Wallis).

Conclusión: Para el grupo de pacientes evaluados no se pudo demostrar una relación entre procesamiento auditivo y percepción asimétrica de la palabra.

Palabras clave: Percepción auditiva, procesamiento auditivo, ventaja del oído derecho, presbiacusia.

\footnotetext{
1 Médico Servicio de Otorrinolaringología Hospital Clínico Universidad de Chile, Departamento de Otorrinolaringología, Facultad de Medicina, Universidad de Chile, Santiago, Chile.

2 Servicio de Otorrinolaringología Hospital Padre Hurtado, Santiago, Chile.

3 Tecnólogo Médico Proyecto Anillo ACT1403, Santiago, Chile.
}

Recibido el 27 de enero, 2018. Aceptado el 11 de marzo, 2018. 


\section{ABSTRACT}

Introduction: Presbycusis corresponds to a symmetrical loss in the audiogram. Nevertheless, some patients complaint that they hear better in one ear.

Aim: The purpose of this work is to analyze if this asymmetrical perception relates with auditory processing.

Material and method: Subjects older than 65 years of age. Exclusion criteria: ear pathology, cognitive decline (Mini Mental test with score <21 points), previous use of hearing aid, asymmetrical thresholds in audiometry. Auditory processing was evaluated with speech in noise test, dichotic tests (dichotic digits and the Spanish version of Staggered spondaic words, SSW), and temporal resolution (frequency pattern and gap in noise). Statistical analysis with SPSS. Descriptive tests, Kruskal Wallis and Mann Whitney. $p<.05$. Protocol approved by the local ethics committee. Subjects consented. Financial support by the Chilean Society of Otorhinolaryngology and Proyecto Anillo ACT1403.

Results: 49 subjects were recruited, 37 female. 19 of them reported better hearing with the right ear, 14 with the left ear, and 16 no difference among ears. Those subjects with symmetrical perception had better PTP than the other two groups. The three groups had no difference in auditory processing evaluation. In the dichotic hearing assessment most patients had a significant better performance for the right ear both for dichotic digits and SSW, independent of the better ear.

Conclusions: For the cases included there was no relation between speech perception and auditory processing. In dichotic tests the right ear had a significant better performance.

Key words: Auditory perception, auditory processing, right ear advantage, presbyacusis.

\section{INTRODUCCIÓN}

De acuerdo a la Organización Mundial de la Salud 360 millones de personas tienen hipoacusia en el mundo, lo que equivale a $5 \%$ de la población mundial ${ }^{1}$. Esto se acentúa en los mayores de 65 años donde se estima que uno de cada tres sufre de hipoacusia ${ }^{2}$. La presbiacusia 0 hipoacusia asociada a la edad corresponde a un déficit auditivo de frecuencias agudas con un patrón audiométrico simétrico para ambos oídos. Sin embargo, en la práctica clínica hay pacientes que dicen escuchar mejor por un oído que por el otro. Esta percepción asimétrica no guarda relación con la función coclear que es posible evaluar mediante la audiometría.

La vía auditiva tiene representación bilateral con múltiples entrecruzamientos entre los estímulos que provienen de cada oído. Sin embargo, hace muchos años que se ha descrito una asimetría en la función auditiva con predominio de los estímulos hablados percibidos por el oído derecho por sobre aquellos percibidos por el oído izquierdo, lo que se ha denominado "ventaja del oído derecho" (VOD) ${ }^{3}$. Diversos estudios han mostrado resultados disímiles en relación a la VOD y envejecimiento 4 . Para algunos autores la ventaja del oído derecho se pierde en adultos mayores ${ }^{5}$ mientras que en otros casos ésta se mantiene ${ }^{3}$.

A raíz de la experiencia clínica de pacientes portadores de hipoacusia simétrica con percepción asimétrica del déficit, surge la pregunta de si esta dicotomía podría tener relación con diferencias en el procesamiento auditivo del estímulo hablado. La hipótesis del presente trabajo es que en pacientes mayores de 65 años portadores de audición simétrica en la audiometría, que perciben un oído como dominante, esto se relaciona con diferencias en pruebas de procesamiento auditivo versus pacientes que tienen percepción simétrica de su déficit. 


\section{MATERIAL Y MÉTODO}

Pacientes. Sujetos mayores de 65 años que cumplan con los siguientes criterios de inclusión y exclusión: sin patología otológica previa, sin antecedente de exposición laboral a ruido, sin entrenamiento musical, examen otoscópico normal, sin deterioro cognitivo evaluado por Mini Mental test (puntaje >21 puntos). A todos los pacientes se les realizó audiometría (125 Hz a 8 $\mathrm{kHz}$ ) e impedanciometría (256 Hz), los sujetos con hipoacusia asimétrica (diferencia de más de $15 \mathrm{~dB}$ $\mathrm{HL}$ en dos frecuencias continuas) y/o patología de oído medio fueron excluidos. En la entrevista inicial se registró si el paciente escucha igual por ambos oídos o percibe dominancia de alguno de ellos.

\section{Evaluación procesamiento auditivo}

Se evaluaron pruebas de:

1. Escucha dicótica:

- Test de habla dicótica: Versión en español de Staggered Spondaic Word Test (SSW-S): corresponde a un test de habla dicótica validado al español ${ }^{6}$. Se presentan 40 grupos de cuatro palabras en forma dicótica a $50 \mathrm{~dB}$ SL en relación con los umbrales de las frecuencias $500,1.000$ y $2.000 \mathrm{~Hz}$. Se obtiene un porcentaje de aciertos por oído.

- Dígitos dicóticos en español: Esta prueba está estandarizada al español ${ }^{7}$ y consiste en presentar en forma dicótica 20 sets de dos pares de dígitos a $50 \mathrm{~dB}$ SL en relación con los umbrales a 500, 1.000 y $2.000 \mathrm{~Hz}^{8}$. Los sujetos deben repetir los sets de cuatro números y se obtiene un resultado correcto por oído. Se obtiene un puntaje por oído.

2. Reconocimiento del habla degradada 0 en ambiente de competencia sonora:

- Versión en español de speech in noise: Prueba validada al español ${ }^{7}$. Se presenta en forma monoaural cuatro randomizaciones de lista de 25 palabras monosilábicas en la presencia de ruido blanco a $40 \mathrm{~dB}$ SL relativo a umbrales tonales de frecuencias $500,1.000$ y $2.000 \mathrm{~Hz}$. Esto se realiza con relación señal/ruido de $10 \mathrm{~dB}$ SNR. LoS sujetos deben repetir cada palabra y se marcan los aciertos.
3. Procesamiento temporal:

- Test de adaptación de resolución temporal (gap detection): El objetivo de este examen es determinar el umbral en milisegundos (ms) de detección de brechas de silencio por parte de un individuo. Para su ejecución se utilizó software The Beta Adult version of the Adaptive Tests of Temporal Resolution ${ }^{\odot}$ (ATTR) desarrollado por Jennifer Lister y Richard Roberts ${ }^{9}$. Este examen emplea un paradigma de respuesta obligada adaptativa ${ }^{10}$. Al sujeto se le presentan dos sonidos. En el sonido standard se incluye un gap de $1 \mathrm{~ms}$ de duración. En el sonido objetivo se incluye un gap de duración adaptativa, es decir, si el sujeto acierta en identificar el sonido objeto la duración del gap disminuye en 1,2 y si el sujeto no acierta aumenta en 1,2. El test finaliza luego de 8 reversas. Los sonidos se presentan en modalidad across channel, es decir, sonido previo al silencio corresponde a ruido de banda ancha centrado en $2 \mathrm{kHz}$, y sonido posterior al silencio centrado en 1 kHz. La duración del burst previo al gap es de $300 \mathrm{~ms}$ y el posterior al gap varía entre 250 y $350 \mathrm{~ms}$.

- Patrón de frecuencia: Prueba monoaural que consiste de 60 secuencias que contienen tres tonos de frecuencia baja $(880 \mathrm{~Hz})$ y alta $(1.122 \mathrm{~Hz})$ en distintas combinaciones. Cada tono tiene una duración de 300 ms $y$ el intervalo entre secuencias es de 6 segundos. El sujeto debe identificar la secuencia.

\section{Aspectos éticos}

El protocolo fue aprobado por el Comité de Ética de Investigación del Hospital Clínico de la Universidad de Chile, y todos los pacientes cuentan con consentimiento informado. Se cauteló que los datos de los pacientes se mantuvieran en forma anónima.

\section{Análisis estadístico}

Se realizó mediante programa SPSS. Se utilizaron estadísticos descriptivos y pruebas no paramédicas (Kruskal Wallis y Mann Whitney). $p<.05$. 


\section{Financiamiento}

Este trabajo cuenta con financiamiento de la Sociedad Chilena de Otorrinolaringología (Concurso de Proyectos de Investigación) y del Proyecto Anillo ACT1403.

\section{RESULTADOS}

Se reclutaron 49 pacientes, 37 de ellos de género femenino. Se identifican tres grupos: escuchan mejor por oído derecho (n: 19), por oído izquierdo (n: 14), o sin diferencia entre ambos oídos (n: 16) (Tabla 1). 17 pacientes tenían PTP (promedio umbral frecuencias $0,5 \mathrm{kHz}$ a $4 \mathrm{kHz}$ ) menor a $20 \mathrm{~dB}$ $\mathrm{HL}, 26$ entre 20 y $40 \mathrm{~dB} \mathrm{HL}$, y 6 entre 40 y $60 \mathrm{~dB}$ HL. El grupo de pacientes con audición simétrica tenía PTP significativamente menor que los otros dos grupos (Tabla 1).

En las pruebas de procesamiento no se encontró diferencia significativa para los grupos en la pruebas de escucha dicótica (dígitos dicóticos y SSW), ni en procesamiento temporal (gap detection y patrón de frecuencia), ni en habla en ruido (Tabla 1).
Al evaluar la VOD en pruebas dicóticas se observó que el oído derecho tuvo un significativo mejor rendimiento que el oído izquierdo para dígitos dicóticos y SSW (p .0 y .007 respectivamente, test de Mann Whitney) en todo el grupo de pacientes evaluados, sin diferencia al agrupar por percepción auditiva ( $p .835$, test de Kruskal Wallis). En particular, el grupo de sujetos que decía escuchar igual por ambos oídos también tuvo mejor rendimiento en oído derecho ( $p .017$, test de Mann Whitney). Los casos de género femenino tuvieron diferencia significativa en relación al género masculino para la prueba de dígitos dicóticos ( $p$.018), sin embargo en la prueba SSW no hubo diferencia entre ambos sexos (p .58).

\section{DISCUSIÓN}

El envejecimiento de la población conlleva un aumento progresivo de pacientes portadores de presbiacusia en la consulta del médico otorrinolaringólogo. En ocasiones es posible que los pacientes refieran escuchar mejor por un oído a pesar de tener umbrales audiométricos simétricos. El presente trabajo busca explicar esta

Tabla 1. Características epidemiológicas de los sujetos según oído predominante. Resultados de pruebas de procesamiento auditivo según oído predominante

\begin{tabular}{|lllll|}
\hline & Bilateral & Oído derecho & Ó́do izquierdo & p \\
\hline Número & 16 & 19 & 14 & \\
Edad promedio (SD) & $72,4(4,6)$ & $73,8(5,1)$ & $76,6(4,4)$ & 0,58 \\
Mujeres (\%) & $12(75 \%)$ & $13(79 \%)$ & $9(71 \%)$ & \\
PTP 0,5-4 KHz & $20(8)$ & $30(10)$ & $28(11)$ & 0,01 \\
Discriminación en ruido 0D & $23(1,2)$ & $22(2,1)$ & $23(1)$ & 0,84 \\
Discriminación en ruido 0I & $23(0,7)$ & $22(2,5)$ & $23(1)$ & 0,26 \\
Dígitos dicóticos 0D & $36(2)$ & $34(6)$ & $34(4)$ & 0,94 \\
Dígitos dicóticos 0I & $28(7)$ & $27(9)$ & $28(6)$ & 0,5 \\
cSSW errores 0D & $3(4)$ & $7(10)$ & $6(7)$ & 0,35 \\
cSSW errores 0I & $5(3)$ & $11(17)$ & $7(5)$ & 0,30 \\
Reconocimiento de frecuencias 0D & $11(7)$ & $10(7)$ & $10(5)$ & 0,85 \\
Reconocimiento de frecuencias 0I & $10(7)$ & $9(6)$ & $9(5)$ & 0,77 \\
GIN AC & $109(69)$ & $125(69)$ & $125(81)$ & 0,78 \\
\hline
\end{tabular}

SD: desviación standard; OD: oído derecho; OI: oído izquierdo; CSSW: pruebas de palabras espondaicas corregida; GIN AC: test de silencio across channel. 
situación a través del estudio del procesamiento auditivo, entendiendo que la audiometría otorga una información limitada de la vía auditiva y su relación con el sistema nervioso central. Para esto se utilizó una batería de exámenes que incluyeron comprensión del habla en competencia (habla en ruido), escucha dicótica (dígitos dicóticos y test de escucha dicótica o SSW) y procesamiento temporal (patrón de frecuencia y discriminación de silencios). Todos estos exámenes en español y previamente validados en Chile.

Los resultados no muestran que haya una correlación entre los parámetros estudiados de procesamiento auditivo y la percepción de audición de los pacientes, ya sea simétrica 0 asimétrica para oído derecho o izquierdo. No encontramos en la literatura reportes similares para poder comparar nuestros resultados, razón por la cual el diseño del método fue exploratorio. Es posible que si se incluye un número mayor de sujetos estos resultados puedan cambiar.

La vía auditiva desde la cóclea hasta la corteza cerebral tienen representación bilateral, sin embargo, el procesamiento de la información de ambos oídos parece no tener la misma relevancia. Para el estímulo hablado la información del oído derecho predomina por sobre el oído izquierdo ${ }^{4}$. Para explicar este fenómeno se han planteado dos teorías. Kimura en el año 1967 describe la teoría estructural: El oído derecho tienen mejor representación en el centro del lenguaje del hemisferio izquierdo, es decir la asimetría sería secundaria a un fenómeno de aferencia de información o "de abajo hacia arriba"11. Estudios posteriores han demostrado que efectivamente la vía auditiva tiene asimetrías tanto periféricas como centrales. Las emisiones otoacústicas espontáneas son más frecuentes en el oído derecho, la amplitud de emisiones otoacústicas transientes son mayores en oído derecho, el tinnitus es más frecuente en el oído izquierdo3. También a nivel central se ha puesto en evidencia que los estímulos verbales captados por el oído derecho predominan, y para los estímulos no verbales predomina la información del oído izquierdo. En el modelo de Kimura también se describe que la información adquirida por el oído izquierdo debe cruzar por el cuerpo calloso hacia el hemisferio derecho lo cual produce un desfase.

La segunda teoría corresponde al modelo por Kinsbourne el año 1978'2. El autor plantea que la asimetría en las funciones de los hemisferios cerebrales sería secundario a un fenómeno atencional 0 "de arriba hacia abajo". Este predominio del oído derecho se ha llamado en la literatura "ventaja del oído derecho". A lo largo de la vida esta asimetría en las funciones auditivas podría modificarse. Algunos autores han demostrado que con el envejecimiento se mantiene la ventaja del oído derecho ${ }^{3,13}$. Sin embargo, otros reportes no coinciden ${ }^{5,14}$. Es importante mencionar que en pruebas de habla dicótica es relevante la atención, por lo tanto, la divergencia en los resultados de dichas publicaciones podría ser secundario a los paradigmas empleados para evaluar los sujetos.

Nuestros resultados muestran que en pacientes mayores de 65 años incluidos en este estudio la ventaja del oído derecho está presente para ambas pruebas de habla dicótica evaluada, dígitos dicóticos y SSW, con una significancia muy importante. Sin embargo, esta observación no tuvo relación con la percepción auditiva del mejor oído. Hubo diferencia por sexo para los resultados de la prueba de dígitos dicóticos, no así para SSW.

\section{CONCLUSIÓN}

La percepción asimétrica de escucha hablada no tuvo relación con características del procesamiento auditivo evaluadas. Se puso en evidencia el fenómeno de ventaja del oído derecho para escucha dicótica en sujetos mayores de 65 años. 


\section{BIBLIOGRAFÍA}

1. http://www.who.int/mediacentre/factsheets/ fs300/en/. Consultado el 24 de enero de 2018.

2. Nienke C. Homans, R. Mick Metselaar, J. Gertuan Dingemanse, Marc P. van der Schroeff, Michael P. Brocaar, Marjan H. Wieringa, et al. Prevalence of Age-Related Hearing Loss, Including Sex Differences, in Older Adults in a Large Cohort Study. Laryngoscope 2016; 127: 725-30.

3. Jerger J, Martin J. Hemispheric asymmetry of the right ear advantage in dichotic listening. Hear Res 2004; 198: 125-36.

4. Martin J, Jerger J. Some effects of aging on central auditory processing. Rehabil Res Dev 2005; 42(4 Suppl 2): 25-44.

5. Bellis TJ, Nicol T, Kraus N. Aging Affects Hemispheric Asymmetry in the Neural Representation of Speech Sounds. J Neurosci 2000; 20: 791-7.

6. Sото, H. \& Windham, R. 1992. El test SSW. Manual de la versión en español. St. Louis: Auditec.

7. Fuente $A, M c P$ herson B. Auditory processing tests for Spanish-speaking adults: An initial study. Int J Audiol 2006; 45: 645-59.

8. Musiek FE. Assessment of central auditory dysfunction: the dichotic digit test revisited. Ear Hear 1983; 4: 79-83.

9. Lister J, Roberts R, Krause J, DeBiase D, Carlson H. An adaptive clinical test of temporal resolution: Within-channel and across-channel gap detection. Int J Audiol 2011; 50: 375-84.

10. LeVItT H. Transformed up-down methods in psychoacoustics. J Acoust Soc Am 1971; 49: Suppl 2: 467.

11. KimURA, D. Functional asymmetry of the brain in dichotic listening. Cortex 1967; 3: 163-78.

12. Kinsbourne, M. 1978. Biological determinants of functional bisymmetry and asymmetry. In: Kinsbourne, M. (Ed.), Asymmetrical Function of the Brain. Cambridge University Press, New York, pp. 3-13.

13. Westerhausen R, Bless J, Kompus K. Behavioral Laterality and Aging: The Free-Recall DichoticListening Right-Ear Advantage Increases With Age. Dev Neuropsychol 2015; 40: 313-27.

14. Vanhoucke E, Cousin E, Baciu M. Hemispheric asymmetry modulation for language processing in aging: meta-analysis of studies using the dichotic listening test. Geriatr Psychol Neuropsychiatr Vieil 2013; 11: 57-64. 